\title{
On a $q$-extension of the linear harmonic oscillator with the continuous orthogonality property on $\mathbb{R}$
}

\author{
R. Álvarez-Nodarse*, M. K. Atakishiyeva†, and N. M. Atakishiyevł \\ * Departamento de Análisis Matemático, Universidad de Sevilla, \\ Apdo. 1160, E-41080 Sevilla, Spain and \\ Instituto Carlos I de Física Teórica y Computacional, \\ Universidad de Granada, E-18071 Granada, Spain \\ E-mail: ran@us.es \\ $†$ Facultad de Ciencias, UAEM, Apartado Postal 396-3, \\ CP 62250, Cuernavaca, Morelos, México \\ E-mail: mesuma@servm.fc.uaem.mx \\ †nstituto de Matemáticas, UNAM, Apartado Postal 273-3, \\ C.P. 62210 Cuernavaca, Morelos, México \\ E-mail: natig@matcuer.unam.mx
}

\begin{abstract}
We discuss a $q$-analogue of the linear harmonic oscillator in quantum mechanics, based on a $q$-extension of the classical Hermite polynomials $H_{n}(x)$, recently introduced by us in [1]. The wave functions in this $q$-model of the quantum harmonic oscillator possess the continuous orthogonality property on the whole real line $\mathbb{R}$ with respect to a positive weight function. A detailed description of the corresponding $q$-system is carried out.
\end{abstract}

\section{Introduction}

In [1] we introduced a $q$-extension of the classical Hermite polynomials $H_{n}(x)$, which satisfy the following requirements: They are polynomials in the variable $x$, which obey a three-term recurrence relation; They are orthogonal on the whole real line $\mathbb{R}$ with respect to a continuous positive weight function; In the limit as $q \rightarrow 1$ they coincide with the Hermite polynomials $H_{n}(x)$. Such a family enables one to build a $q$-deformed version of the linear harmonic oscillator in quantum mechanics, which is still defined on the whole real line $\mathbb{R}$ and enjoys the continuous orthogonality property on $\mathbb{R}$ with respect to a positive weight function. Let us point out here that there are several publications (see [2]-[10] and references therein) devoted to the study of explicit realizations, which represent $q$-extensions of the Hermite functions (or the wave functions of the linear harmonic oscillator) $H_{n}(x) e^{-x^{2} / 2}$. But none of these realizations satisfies all of the aforementioned requirements: the continuous weight functions in $[2,4,7]$ are supported on the finite intervals; the continuous weight functions in $[3,8]$ are not positive; the $q$-extensions in [2], [4]-[9] are not expressed in terms of polynomials in the independent variable; and, finally, the orthogonality relations in [5]-[7], [10] are discrete.

Our main goal in this paper has been to employ this q-extension of the Hermite polynomials, $\mathcal{H}_{n}(x ; q)$, in order to built a $q$-analogue to the linear harmonic oscillator in quantum mechanics. Section 2 collects those known results from [1] about the polynomials $\mathcal{H}_{n}(x ; q)$, which are needed in section 3 to derive an explicit form of the wave functions $\psi_{n}(x ; q)$ in this $q$-model and their properties. Section 4 is devoted to explicit construction of the generators of the dynamical symmetry algebra $s u_{q}(1,1)$ in terms of the lowering and raising $q$-difference operators $a(x ; q)$ and $a^{\dagger}(x ; q)$. Concluding section 5 contains a brief discussion of $q$-coherent states for this $q$-extension of the quantum harmonic oscillator. 


\section{Definition and properties of the polynomials $\mathcal{H}_{n}(x ; q)$}

In [1] the following family was introduced

$$
\begin{gathered}
\mathcal{H}_{2 n}(x ; q):=(-1)^{n}(q ; q)_{n} L_{n}^{(-1 / 2)}\left(x^{2} ; q\right) \\
=(-1)^{n}\left(q^{1 / 2} ; q\right)_{n 1} \phi_{1}\left(\begin{array}{c}
q^{-n} \\
q^{1 / 2}
\end{array} \mid q ;-q^{n+1 / 2} x^{2}\right)=(-1)^{n}{ }_{2} \phi_{1}\left(\begin{array}{c}
q^{-n},-x^{2} \\
0
\end{array} \mid q ; q^{n+1 / 2}\right), \\
\mathcal{H}_{2 n+1}(x ; q):=(-1)^{n}(q ; q)_{n} x L_{n}^{(1 / 2)}\left(x^{2} ; q\right) \\
=(-1)^{n}\left(q^{3 / 2} ; q\right)_{n} x_{1} \phi_{1}\left(\begin{array}{c}
q^{-n} \\
q^{3 / 2}
\end{array} \mid q ;-q^{n+3 / 2} x^{2}\right)=(-1)^{n} x_{2} \phi_{1}\left(\begin{array}{c}
q^{-n},-x^{2} \\
0
\end{array} \mid q ; q^{n+3 / 2}\right),
\end{gathered}
$$

where $L_{n}^{(\alpha)}(x ; q)$ are $q$-Laguerre polynomials, ${ }_{1} \phi_{1}$ and ${ }_{2} \phi_{1}$ denote the basic hypergeometric polynomials and $(a ; q)_{n}$ is the $q$-shifted factorial (we employ standard notations of $q$-analysis, see, for example, [11] or [12]). In (2.1) and throughout the sequel it is assumed that $q$ is a fixed number such that $0<q<1$.

This family is generated by the three-term recurrence relation

$$
x \mathcal{H}_{n}(x ; q)=q^{-n / 2} \mathcal{H}_{n+1}(x ; q)-\left(1-q^{-n / 2}\right) \mathcal{H}_{n-1}(x ; q), \quad n=0,1,2, \ldots,
$$

with the initial condition $\mathcal{H}_{0}(x ; q) \equiv 1$.

The polynomials (2.1) satisfy the continuous orthogonality relation

$$
\int_{-\infty}^{\infty} \mathcal{H}_{m}(x ; q) \mathcal{H}_{n}(x ; q) \frac{d x}{E_{q}\left(x^{2}\right)}=\pi q^{-n / 2}\left(q^{1 / 2} ; q^{1 / 2}\right)_{n}\left(q^{1 / 2} ; q\right)_{1 / 2} \delta_{m n}
$$

on the whole real line $\mathbb{R}$ with respect to the positive weight function $w(x)=1 / E_{q}\left(x^{2}\right)=$ $1 /\left(-x^{2} ; q\right)_{\infty}[1]$

The polynomials $\mathcal{H}_{n}(x ; q)$ constitute a $q$-extension of the classical Hermite polynomials $H_{n}(x)$ since these polynomials reduce to the latter in the limit as $q \rightarrow 1$, i.e.,

$$
\lim _{q \rightarrow 1}(1-q)^{-n / 2} \mathcal{H}_{n}(\sqrt{1-q} x ; q)=2^{-n} H_{n}(x),
$$

From the recurrence relation $(2.2)$ it follows that the $\mathcal{H}_{n}(x ; q)$ can be expressed in terms of the discrete $q$-Hermite polynomials $\tilde{h}(x ; q)$ of type II as

$$
\mathcal{H}_{n}\left(x ; q^{2}\right)=q^{n(n-1) / 2} \tilde{h}_{n}(x ; q):=i^{-n}{ }_{2} \phi_{0}\left(\begin{array}{c}
q^{-n}, i x \\
-
\end{array} \mid q ;-q^{n}\right) .
$$

So from the known $q$-difference equation for the discrete $q$-Hermite polynomials $\tilde{h}_{n}(x ; q)$ (see [13], (3.29.5), p.119) one deduces that

$$
\begin{aligned}
& \left(1-q^{n / 2}\right) x^{2} \mathcal{H}_{n}(x ; q)=\left(1+q^{1 / 2}+x^{2}\right) \mathcal{H}_{n}(x ; q) \\
& -\left(1+x^{2}\right) \mathcal{H}_{n}\left(q^{1 / 2} x ; q\right)-q^{1 / 2} \mathcal{H}_{n}\left(q^{-1 / 2} x ; q\right) .
\end{aligned}
$$

Similarly, one readily verifies that the forward and backward shift operators for the polynomials $\mathcal{H}_{n}(x ; q)$ are of the form

$$
\begin{aligned}
& {\left[q^{-\frac{1}{2} x \frac{d}{d x}}-1\right] \mathcal{H}_{n}(x ; q)=q^{-1 / 2}\left(1-q^{n / 2}\right) x \mathcal{H}_{n-1}(x ; q),} \\
& {\left[\left(1+x^{2}\right) q^{\frac{1}{2} x \frac{d}{d x}}-1\right] \mathcal{H}_{n}(x ; q)=x \mathcal{H}_{n+1}(x ; q),}
\end{aligned}
$$


respectively, where $q^{a x \frac{d}{d x}}$ is the dilation operator, i.e., $q^{a x \frac{d}{d x}} f(x)=f\left(q^{a} x\right)$.

A Rodrigues-type difference formula for the polynomials $\mathcal{H}_{n}(x ; q)$ can be written as

$$
\mathcal{H}_{n}(x ; q)=(-x)^{-n} E_{q}\left(x^{2}\right)\left(q^{\frac{1}{2} x \frac{d}{d x}} ; q^{-1 / 2}\right)_{n} E_{q}^{-1}\left(x^{2}\right),
$$

where we have slightly simplified the $n$-th power of the $q$-derivative operator $\mathcal{D}_{q}(c f(3.29 .10)$ in [13], p.119) by representing it in the form

$$
\mathcal{D}_{q}^{n} \equiv \frac{1}{(1-q)^{n} x^{n}}\left(q^{x \frac{d}{d x}} ; q^{-1}\right)_{n}, \quad n=0,1,2, \ldots .
$$

It is not difficult to prove (2.9) by induction on the power $n$.

Finally, using the generation function for the discrete $q$-Hermite polynomials $\tilde{h}_{n}(x ; q)$ of type II [13], one finds that

$$
\frac{\left(-x t ; q^{1 / 2}\right)_{\infty}}{\left(-t^{2} ; q\right)_{\infty}}=\sum_{n=0}^{\infty} \frac{1}{\left(q^{1 / 2} ; q^{1 / 2}\right)_{n}} \mathcal{H}_{n}(x ; q) t^{n} .
$$

\section{Wave functions $\psi_{n}(x ; q)$ and their properties}

We wish to discuss a $q$-model of the linear harmonic oscillator, which is described by the wave functions of the form

$$
\psi_{n}(x ; q):=d_{n}^{-1}(q) \mathcal{H}_{n}(x ; q) E_{q}^{-1 / 2}\left(x^{2}\right)
$$

with the normalization constant $d_{n}(q):=q^{-n / 4} \sqrt{\pi\left(q^{1 / 2} ; q\right)_{1 / 2}\left(q^{1 / 2} ; q^{1 / 2}\right)_{n}}$. Then, by continuous orthogonality relation $(2.3)$, these functions are orthonormal on $\mathbb{R}$, that is,

$$
\int_{-\infty}^{\infty} \psi_{m}(x ; q) \psi_{n}(x ; q) d x=\delta_{m n} .
$$

The wave functions $\psi_{n}(x ; q)$ are defined by (3.1) in such a way that in the limit as $q \rightarrow 1$ they coincide with the orthonormalized Hermite functions (or the wave functions of the linear harmonic oscillator in non-relativistic quantum mechanics):

$$
\lim _{q \rightarrow 1} \psi_{n}(\sqrt{1-q} \xi ; q)=\frac{1}{\sqrt{\sqrt{\pi} 2^{n} n !}} H_{n}(\xi) \exp \left(-\xi^{2} / 2\right)=: \psi_{n}(\xi)
$$

This limit property of $\psi_{n}(x ; q)$ follows immediately from (2.4) and the well-known fact

$$
\lim _{q \rightarrow 1} E_{q}((1-q) z)=e^{z}
$$

about the Jackson $q$-exponential function $E_{q}(z)$ (see [11] or [12]).

From (2.6) and (3.1) one obtains that the wave functions $\psi_{n}(x ; q)$ are eigenfunctions of the $q$-Hamiltonian $H(x ; q)$,

$$
H(x ; q) \psi_{n}(x ; q)=E_{n}(q) \psi_{n}(x ; q), \quad E_{n}(q):=\frac{1-q^{n / 2}}{1-q^{1 / 2}} .
$$

By equation (2.6), the explicit form of this self-adjoint $q$-difference operator is

$$
H(x ; q):=\frac{1}{\left(1-q^{1 / 2}\right) x^{2}}\left[\left(1+x^{2}+q^{1 / 2}\right) I-\sqrt{1+x^{2}} q^{\frac{1}{2} x \frac{d}{d x}}-q^{\frac{1}{2}\left(1-x \frac{d}{d x}\right)} \sqrt{1+x^{2}}\right],
$$


where $I$ is the identity operator. This expression for $H(x ; q)$ in terms of the dilation operators $q^{ \pm \frac{1}{2} x \frac{d}{d x}}$ may create an impression that the $H(x ; q)$ contains singularity at $x=0$ due to the presence of the factor $x^{2}$ in the denominator. To remove this doubt one should take into account that, by definition (3.6),

$$
\begin{gathered}
H(x ; q) \psi_{n}(x ; q)=\frac{1}{(1-\sqrt{q}) x^{2}}\left[\left(1+\sqrt{q}+x^{2}\right) \psi_{n}(x ; q)\right. \\
\left.-\sqrt{1+x^{2}} \psi_{n}\left(q^{1 / 2} x ; q\right)-\sqrt{q+x^{2}} \psi_{n}\left(q^{-1 / 2} x ; q\right)\right]
\end{gathered}
$$

for all $n=0,1,2, \ldots$. Besides, from (3.1) it is evident that the wave functions $\psi_{n}(x ; q)$ have regular behavior around $x=0$. Now substituting the sum of first two terms $c_{0}+c_{1} x$ from the expansion of $\psi_{n}(x ; q)$ around $x=0$ into expression in square brackets in (3.7) and keeping only constant and linear in $x$ terms, one readily verifies that

$$
(1+\sqrt{q})\left(c_{0}+c_{1} x\right)-\left(c_{0}+c_{1} \sqrt{q} x\right)-\sqrt{q}\left(c_{0}+\frac{c_{1}}{\sqrt{q}} x\right)=0 .
$$

Consequently, the total combination inside the square brackets in (3.7) behaves like $x^{2}$ in the $x \rightarrow 0$ limit and the right side of (3.7) therefore assumes a constant value at $x=0$. This confirms that there is no singularity at $x=0$.

We observe also that the eigenvalues $E_{n}(q)$ of $H(x ; q)$ are bounded from above by the asymptotic value $E_{\infty}(q)=1 /\left(1-q^{1 / 2}\right)$ and, since $E_{n+1}(q)-E_{n}(q)=q^{n / 2}$, they are not equidistant.

From $(2.2)$ it follows that the wave functions $\psi_{n}(x ; q)$ satisfy the three-term recurrence relation

$$
x \psi_{n}(x ; q)=q^{-(2 n+1) / 4} \sqrt{1-q^{(n+1) / 2}} \psi_{n+1}(x ; q)+q^{(1-2 n) / 4} \sqrt{1-q^{n / 2}} \psi_{n-1}(x ; q)
$$

with the initial condition that the ground state $\psi_{0}(x ; q)=d_{0}^{-1}(q) E_{q}^{-1 / 2}\left(x^{2}\right)$.

Likewise, from the explicit form of the forward and backward shift operators (2.7) it follows that

$$
a(x ; q) \psi_{n}(x ; q)=\sqrt{E_{n}(q)} \psi_{n-1}(x ; q), \quad a^{\dagger}(x ; q) \psi_{n}(x ; q)=\sqrt{E_{n+1}(q)} \psi_{n+1}(x ; q),
$$

where the $q$-difference lowering and raising operators $a(x ; q)$ and $a^{\dagger}(x ; q)$ are given by

$$
\begin{aligned}
& a(x ; q)=\frac{q^{1 / 4}}{\sqrt{1-q^{1 / 2}} x}\left(q^{-\frac{1}{2} x \frac{d}{d x}} \sqrt{1+x^{2}}-I\right), \\
& a^{\dagger}(x ; q)=\frac{q^{1 / 4}}{\sqrt{1-q^{1 / 2}} x}\left(\sqrt{1+x^{2}} q^{\frac{1}{2} x \frac{d}{d x}}-I\right),
\end{aligned}
$$

respectively. We invite the reader to verify that these operators are indeed mutually adjoint in the Hilbert space $L^{2}(\mathbb{R}, d x)$ of square integrable functions $f(x)$ with respect to $d x$.

Similar to the case of the quantum linear harmonic oscillator, the lowering and raising operators (3.10) factorize the Hamiltonian (3.6), that is,

$$
H(x ; q)=a^{\dagger}(x ; q) a(x ; q) .
$$

Moreover, it is not difficult to verify, by using (3.10), that their another (i.e., when the operator $a(x ; q)$ is right multiplied by its adjoint operator $\left.a^{\dagger}(x ; q)\right)$ product $a(x ; q) a^{\dagger}(x ; q)$ 
is equal to $I+q^{1 / 2} H(x ; q)$. This means that the operators $a(x ; q)$ and $a^{\dagger}(x ; q)$ satisfy the $q$-commutation relation of the form

$$
a(x ; q) a^{\dagger}(x ; q)-q^{1 / 2} a^{\dagger}(x ; q) a(x ; q) \equiv\left[a(x ; q), a^{\dagger}(x ; q)\right]_{q^{1 / 2}}=I .
$$

It should be noted at this point that we have used above the known explicit form of the forward and backward shift operators $(2.7)$ for the polynomials $\mathcal{H}_{n}(x ; q)$ in order to find the lowering and raising operators $a(x ; q)$ and $a^{\dagger}(x ; q)$. But we could have started equivalently with the $q$-difference equation (3.5) itself and have directly factorized it in terms of the same operators $a(x ; q)$ and $a^{\dagger}(x ; q)$ (for a more detailed discussion of the factorization of difference equations, see, for example, $[15,16])$.

So we have established that our $q$-model is governed by the Hamiltonian (3.6), which admits the factorization (3.11) in terms of the operators $a(x ; q)$ and $a^{\dagger}(x ; q)$, satisfying the $q$ commutation relation (3.12). This characteristic property of the Hamiltonian (3.6) is known to reflect the fact that the dynamical symmetry of this $q$-model is described by the quantum algebra $s u_{q}(1,1)[14]$. In the next section we construct explicitly the generators of this algebra in terms of the lowering and raising operators $a(x ; q)$ and $a^{\dagger}(x ; q)$.

\section{Dynamical symmetry}

In this section we remind the reader first how one constructs a dynamical symmetry algebra for the linear harmonic oscillator, which is governed in non-relativistic quantum mechanics by the well-known Hamiltonian

$$
H(x):=\frac{\hbar \omega}{2}\left(\xi^{2}-\frac{d^{2}}{d \xi^{2}}\right) \equiv \hbar \omega\left(N(x)+\frac{1}{2}\right),
$$

where $\xi=\sqrt{m w / \hbar} x$ is a dimensionless coordinate, $N(x)$ is the particle number operator,

$$
N(x):=a^{\dagger}(x) a(x),
$$

and the annihilation and creation operators are defined as usual:

$$
\begin{gathered}
a(x)=\frac{1}{\sqrt{2}}\left(\xi+\frac{d}{d \xi}\right), \quad a^{\dagger}(x)=\frac{1}{\sqrt{2}}\left(\xi-\frac{d}{d \xi}\right), \\
{\left[a(x), a^{\dagger}(x)\right] \equiv a(x) a^{\dagger}(x)-a^{\dagger}(x) a(x)=I .}
\end{gathered}
$$

By using (4.1) and (4.3) one readily verifies that

$$
[H(x), a(x)]=-a(x), \quad\left[H(x), a^{\dagger}(x)\right]=a^{\dagger}(x) .
$$

Observe that in the case of the linear harmonic oscillator (4.1) there is no much difference between the Hamiltonian $H(x)$ and the particle number operator $N(x)$ : the former operator, divided by the factor $\hbar \omega$, is equal to the latter one plus a constant term $1 / 2$. So, the particle number operator $N(x)$ satisfies the same commutation relations (4.4) with the annihilation and creation operators $a(x)$ and $a^{\dagger}(x)$.

Having factorized the Hamiltonian $H(x)$ (or, equivalently, the particle number operator $N(x)$ ) in terms of the annihilation $a(x)$ and creation $a^{\dagger}(x)$ operators, one explicitly constructs the closed Lie algebra $s u(1,1)$ with the three generators

$$
K_{0}(x):=\frac{1}{2 \hbar \omega} H(x) \equiv \frac{1}{2}\left(N(x)+\frac{1}{2}\right), \quad K_{+}(x):=\frac{1}{2}\left(a^{\dagger}(x)\right)^{2}, \quad K_{-}(x):=\frac{1}{2} a^{2}(x) .
$$


Indeed, it is not difficult to verify that thus defined generators satisfy the standard commutation relations

$$
\left[K_{0}(x), K_{ \pm}(x)\right]= \pm K_{ \pm}(x), \quad\left[K_{-}(x), K_{+}(x)\right]=2 K_{0}(x),
$$

of the algebra $s u(1,1)$. Unitary irreducible representations of this algebra are known to be characterized by eigenvalues of the invariant (that is, commuting with all three generators (4.5)) Casimir operator

$$
C:=K_{0}(x)\left[K_{0}(x)-I\right]-K_{+}(x) K_{-}(x)=s(s-1) I .
$$

A direct calculation of the Casimir operator (4.7) with the aid of (4.5) shows that the eigenvalue $s(s-1)$ in this particular case is equal to $-3 / 16$. This means that the parameter $s$ may be equal to either $s_{1}=1 / 4$ or $s_{2}=3 / 4$. Each of these two values of $s$ defines a unitary irreducible representation of the algebra $s u(1,1): D^{+}(1 / 4)$ consists of those eigenstates of the Hamiltonian $H(x)$, which correspond to the eigenvalues $s_{1}+n=n+1 / 4=(2 n+1 / 2) / 2$, $n=0,1,2, \ldots$, of the generator $K_{0}(x)=H(x) / 2 \hbar \omega$; whereas $D^{+}(3 / 4)$ corresponds to the eigenvalues $s_{2}+n=n+3 / 4=(2 n+1+1 / 2) / 2$ of the same generator $K_{0}(x)$. So in this way one arrives at the correct spectrum $E_{n}=\hbar \omega(n+1 / 2)$ of the Hamiltonian $H(x)$, without solving an eigenvalue problem for the appropriate Schrödinger equation. Thus eigenstates of $H(x)$ with the eigenvalues $E_{2 n}$ form the unitary irreducible representation $D^{+}(1 / 4)$ and those with $E_{2 n+1}$ form another one, $D^{+}(3 / 4)$.

The Fock space $\mathcal{H}_{F}$ of all eigenfunctions $\left\{\psi_{n}(x)\right\}$ of the Hamiltonian $H(x)$ splits into two $s u(1,1)$-irreducible subspaces for $H(x)$ is symmetric with respect to the inversion $x \rightarrow-x$. Therefore the inversion operator $P, P x=-x$, commutes with all three generators (4.5) and $\mathcal{H}_{F}$ decomposes into two irreducible components,

$$
\mathcal{H}_{F}=\mathcal{H}_{0} \oplus \mathcal{H}_{1}
$$

consisting of the wave functions $\psi_{n}(x)$ with even and odd indices $n$, respectively. The irreducible subspaces $\mathcal{H}_{0}$ and $\mathcal{H}_{1}$ are characterized by the eigenvalues $(-1)^{\epsilon}$ of the operator $P$ with $\epsilon=0$ in $\mathcal{H}_{0}$ and $\epsilon=1$ in $\mathcal{H}_{1}$. It is clear that the subspaces $\mathcal{H}_{0}$ and $\mathcal{H}_{1}$ correspond to the unitary irreducible representations $D^{+}(1 / 4)$ and $D^{+}(3 / 4)$, respectively.

Now we are in a position to discuss a dynamical symmetry algebra for the $q$-model (3.1). To construct it one needs to introduce first the operator [14]

$$
N(x ; q):=\frac{2}{\ln q} \ln \left[1-\left(1-q^{1 / 2}\right) H(x ; q)\right] .
$$

Since the wave functions $\psi_{n}(x ; q)$ are eigenfunctions of the $q$-Hamiltonian $H_{n}(x ; q)$ with the eigenvalues $E_{n}=\left(1-q^{n / 2}\right) /\left(1-q^{1 / 2}\right)$, from the definition (4.9) one deduces that

$$
N(x ; q) \psi_{n}(x ; q)=n \psi_{n}(x ; q),
$$

that is, $N(x ; q)$ is the particle number operator and

$$
[N(x ; q), a(x ; q)]=-a(x ; q), \quad\left[N(x ; q), a^{\dagger}(x ; q)\right]=a^{\dagger}(x ; q) .
$$

At the next step one defines a new set of the operators

$$
b(x ; q):=q^{-N(x ; q) / 8} a(x ; q), \quad b^{\dagger}(x ; q):=a^{\dagger}(x ; q) q^{-N(x ; q) / 8},
$$

which satisfy, according to (4.11), the following commutation relation

$$
b(x ; q) b^{\dagger}(x ; q)-q^{1 / 4} b^{\dagger}(x ; q) b(x ; q)=q^{-N(x ; q) / 4} .
$$


This is readily verified with the aid of (4.11). The operators $b(x ; q), b^{\dagger}(x ; q)$, and $N(x ; q)$ directly lead to the dynamical algebra $s u_{q^{1 / 2}}(1,1)$ with the generators

$$
\begin{gathered}
K_{+}(x ; q):=\gamma\left(b^{\dagger}(x ; q)\right)^{2}, \quad K_{-}(x ; q):=\gamma b^{2}(x ; q), \quad K_{0}(x ; q):=\frac{1}{2}\left(N(x ; q)+\frac{1}{2}\right), \\
\gamma=[1 / 2]_{q^{1 / 2}} .
\end{gathered}
$$

It is not difficult to check that thus defined generators (4.14) satisfy the standard commutation relations

$$
\left[K_{0}(x ; q), K_{ \pm}(x ; q)\right]= \pm K_{ \pm}(x ; q), \quad\left[K_{-}(x ; q), K_{+}(x ; q)\right]=\left[2 K_{0}(x ; q)\right]_{q^{1 / 2}}
$$

of the quantum algebra $s u_{q^{1 / 2}}(1,1)$. The $q$-number $[A]_{q}$ in $(4.14)$ is given by the common expression

$$
[A]_{q}:=\frac{q^{A}-q^{-A}}{q-q^{-1}} .
$$

We are interested in the positive discrete series representations of the quantum algebra $s u_{q}(1,1)$ with lowest weights. These irreducible representations of $s u_{q}(1,1)$ are denoted by $T_{l}^{+}$, where $l$ is the lowest weight, which can be any positive number (see, for example, [17]). It is the characteristic property of every $T_{l}^{+}$that the generator $K_{0}(x ; q)$ has the eigenvalues $l+n, n=0,1,2, \ldots$, in $T_{l}^{+}$.

The invariant Casimir operator in the case under discussion is equal to

$$
C(q):=\left[K_{0}(x ; q)-1 / 2\right]_{q^{1 / 2}}^{2}-K_{+}(x ; q) K_{-}(x ; q)-\frac{1}{4} I=\left([1 / 4]_{q^{1 / 2}}^{2}-1 / 4\right) I .
$$

This means that two possible values of the parameter $s$ in this case are

$$
s_{1}(q)=1 / 2-[1 / 4]_{q^{1 / 2}}, \quad s_{2}(q)=1 / 2+[1 / 4]_{q^{1 / 2}} .
$$

Since $[a]_{q} \rightarrow a$ in the limit as $q \rightarrow 1$ by definition of the $q$-number (4.16), the eigenvalue of $C(q)$ in (4.17) reduces in this limit to the eigenvalue for the Casimir operator in the case of the linear harmonic oscillator (4.7). Evidently, the same happens with the values of $s_{1}(q)$ and $s_{2}(q)$ : they coincide in this limit with the corresponding values of the parameter $s$ in (4.7), i.e.,

$$
\lim _{q \rightarrow 1} s_{1}(q)=\frac{1}{4}, \quad \lim _{q \rightarrow 1} s_{2}(q)=\frac{3}{4} .
$$

From (4.17) it now follows that the lowest weights in our case are $1 / 4$ and $3 / 4$. Therefore by (4.14) the eigenvalues of the particle number operator $N(x ; q) \equiv 2 K_{0}(x ; q)-1 / 2$ are equal to $2 n$ and $2 n+1, n=0,1,2, \ldots$, respectively. Taking into account interrelation (4.9) between the operators $N(x ; q)$ and $H(x ; q)$, one thus arrives at the correct spectrum (3.5) for the Hamiltonian $H(x ; q)$, without solving an eigenvalue problem for $H(x ; q)$.

So we conclude that the wave functions $\psi_{n}(x ; q)$, defined in (3.1), form a representation of the quantum algebra $s u_{q^{1 / 2}}(1,1)$ in the Fock space $\mathcal{H}_{F}$. This representation in the space $\mathcal{H}_{F}$ is reducible precisely for the same reason as in the case of the linear harmonic oscillator (4.1). Thus $\mathcal{H}_{F}$ splits into two $s u_{q^{1 / 2}}(1,1)$-irreducible subspaces $\mathcal{H}_{0} \equiv T_{1 / 4}^{+}$and $\mathcal{H}_{1} \equiv T_{3 / 4}^{+}$, consisting of the wave functions $\psi_{n}(x ; q)$ with even and odd indices $n$, respectively. 


\section{$5 \quad q$-coherent states}

As in the case of the non-relativistic linear harmonic oscillator, one can construct $q$-coherent states for this model as eigenfunctions of the lowering operator $a(x ; q)$, that is,

$$
a(x ; q) \varphi_{\zeta}(x ; q)=\zeta \varphi_{\zeta}(x ; q),
$$

where $\zeta$ is some arbitrary number. To find an explicit form of these states $\varphi_{\zeta}(x ; q)$, we first note that by (3.8)

$$
\psi_{n}(x ; q)=c_{n}(q)\left[a^{\dagger}(x ; q)\right]^{n} \psi_{0}(x ; q), \quad c_{n}(q):=\sqrt{\frac{\left(1-q^{1 / 2}\right)^{n}}{\left(q^{1 / 2} ; q^{1 / 2}\right)_{n}}} .
$$

Consequently, with the aid of (3.8) it is not difficult to verify that the states

$$
\varphi_{\zeta}(x ; q):=f_{q}(\zeta) \sum_{n=0}^{\infty} c_{n}(q) \zeta^{n} \psi_{n}(x ; q),
$$

where $f_{q}(\zeta)$ is some normalization factor (see below), are indeed the eigenstates of the operator $a(x ; q)$ with the eigenvalues $\zeta$. They form an overcomplete system in the Hilbert space $\mathcal{H}_{F}$ and they are not orthogonal in this space. In fact, one can prove, by using expansion (5.3) and orthogonality relation (3.2), that

$$
\int_{-\infty}^{\infty} \varphi_{\zeta}(x ; q) \varphi_{\zeta^{\prime}}(x ; q) d x=f_{q}(\zeta) f_{q}\left(\zeta^{\prime}\right) e_{q^{1 / 2}}\left(\left(1-q^{1 / 2}\right) \zeta \zeta^{\prime}\right)
$$

where

$$
e_{q}(z):=\sum_{n=0}^{\infty} \frac{z^{n}}{(q ; q)_{n}}=\frac{1}{(z ; q)_{\infty}}, \quad|z|<1 .
$$

The normalization condition that the integral on the left of (5.4) is equal to 1 requires to choose $f_{q}(\zeta)=\sqrt{E_{q^{1 / 2}}\left(-\left(1-q^{1 / 2}\right) \zeta^{2}\right)}$. Thus,

$$
\varphi_{\zeta}(x ; q)=\sqrt{E_{q^{1 / 2}}\left(-\left(1-q^{1 / 2}\right) \zeta^{2}\right)} \sum_{n=0}^{\infty} c_{n}(q) \zeta^{n} \psi_{n}(x ; q),
$$

Substitute now into expansion (5.5) explicit form of the coefficients $c_{n}(q)$ from (5.2) and the normalization constants $d_{n}(q)$ for the wave functions $\psi_{n}(x ; q)$ from (3.1) and employ then the generating function (2.10) for the polynomials $\mathcal{H}_{n}(x ; q)$. This yields the final form of the normalized $q$-coherent eigenfunctions of the lowering operator $a(x ; q)$ :

$$
\varphi_{\zeta}(x ; q)=\sqrt{\frac{E_{q^{1 / 2}}\left(-\left(1-q^{1 / 2}\right) \zeta^{2}\right)}{\pi\left(q^{1 / 2} ; q\right)_{1 / 2} E_{q}\left(x^{2}\right)}} \frac{E_{q^{1 / 2}}\left(q^{1 / 4} \sqrt{1-q^{1 / 2}} x \zeta\right)}{E_{q}\left(q^{1 / 2}\left(1-q^{1 / 2}\right) \zeta^{2}\right)} .
$$

Acknowledgments: The research of RAN has been partially supported by the DGES grant BFM 2003-06335-C03-01 and PAI grant FQM-0262. The participation of NMA in this work has been supported in part by the UNAM-DGAPA grant IN102603-3 Óptica Matemática. The main part of this work was performed during a visit by NMA to the Facultad de Matemáticas, Universidad de Sevilla, in June, 2004; he gratefully acknowledges the support for this visit by the Junta Andalucía, grant 2003. 


\section{References}

[1] R. Álvarez-Nodarse, M. K. Atakishiyeva, and N. M. Atakishiyev. On a $q$-extension of the Hermite polynomials $H_{n}(x)$ with the continuous orthogonality property on $\mathbb{R}$. Boletín de la Sociedad Matemática Mexicana (3), 8, No.2, pp.127-139, 2002.

[2] A. J. Macfarlane. On $q$-analogues of the quantum harmonic oscillator and the quantum group $S U(2)_{q}$. J. Phys. A: Math. Gen., 22, No.21, pp. 4581-4588, 1989.

[3] E. D. Kagramanov, R. M. Mir-Kasimov, and Sh. M. Nagiyev. The covariant linear oscillator and generalized realization of the dynamical $S U(1,1)$ symmetry algebra. J. Math. Phys., 31, No.7, pp.1733-1738, 1990.

[4] N. M. Atakishiyev and S. K. Suslov. Difference analogs of the harmonic oscillator. Theor. Math. Phys., 85, No.1, pp.1055-1062, 1991.

[5] N. M. Atakishiyev and S. K. Suslov. A realization of the $q$-harmonic oscillator. Theor. Math. Phys., 87, No.1, pp.442-444, 1991.

[6] R. Askey and S. K. Suslov. The $q$-harmonic oscillator and an analogue of the Charlier polynomials. J. Phys. A: Math. Gen., 26, No.15, pp.L693-L698, 1993.

[7] R. Askey and S. K. Suslov. The $q$-harmonic oscillator and the Al-Salam and Carlitz polynomials. Lett. Math. Phys., 29, No.2, pp.123-132, 1993.

[8] N. M. Atakishiyev and Sh. M. Nagiyev. On the wave functions of a covariant linear oscillator. Theor. Math. Phys., 98, No.2, pp.162-166, 1994.

[9] N. M. Atakishiyev, A. Frank, and K. B. Wolf. A simple difference realization of the Heisenberg q-algebra. J. Math. Phys., 35, No.7, pp.3253-3260, 1994.

[10] R. Hinterding and J. Wess. q-deformed Hermite polynomials in q-quantum mechanics. Eur. Phys. J. C, 6, No.1, pp.183-186, 1999.

[11] G. Gasper and M. Rahman. Basic Hypergeometric Series, Cambridge University Press, Cambridge, 1990.

[12] G. E. Andrews, R. Askey, and R. Roy. Special Functions, Cambridge University Press, Cambridge, 1999.

[13] R. Koekoek and R. F. Swarttouw. The Askey-scheme of hypergeometric orthogonal polynomials and its $q$-analogue, Report 98-17, Delft University of Technology, Delft, 1998.

[14] P. P. Kulish and E. V. Damaskinsky. On the $q$-oscillator and the quantum algebra $s u_{q}(1,1)$. J. Phys.A: Math. Gen., 23, No.9, pp.L415-L419, 1990.

[15] G. Bangerezako. Discrete Darboux transformation for discrete polynomials of hypergeometric type. J. Phys. A: Math. Gen., 31, No.9, pp.2191-2196, 1998.

[16] R. Álvarez-Nodarse, N. M. Atakishiyev, and R. S. Costas-Santos. Factorization of the hypergeometic-type difference equation on non-uniform lattices: dynamical algebra. J. Phys. A: Math. Gen., 38, No.1, pp.152-174, 2005.

[17] I. M. Burban and A. U. Klimyk. Representations of the quantum algebra $U_{q}\left(s u_{1,1}\right)$. J. Phys. A: Math. Gen., 26, No.9, pp.2139-2151, 1993. 